\title{
HiCrypt: A Specialized Translator for Symmetric Block Cipher and GPGPU
}

\author{
Keisuke IWAI $^{\dagger a}$, Naoki NISHIKAWA ${ }^{\dagger}$, and Takakazu KUROKAWA $^{\dagger}$, Members $^{\circ}$
}

\begin{abstract}
SUMMARY Many-core computer systems with GPUs are coming into mainstream use from high-end computing, including supercomputers, to embedded processors. Consequently, the implementation of cryptographic methods on GPGPU is also becoming popular because of such systems' performance. However, many factors affect the performance of GPUs. To cope with this problem, we developed a new translator, HiCrypt, which can generate an optimized GPGPU program written in both of CUDA and OpenCL from a cipher program written in standard C language with directives. Users must annotate only variables and an encoding/decoding function, which are characteristics of cipher programs, with directives. To evaluate the translator, five representative cipher programs are translated into CUDA and OpenCL programs by the translator. Generated programs perform high throughput almost identical to hand optimized programs for all five cipher programs. HiCrypt will contribute to development and evaluate of new and various symmetric block ciphers using GPGPU.

key words: translator, CUDA, OpenCL, symmetric block ciphers, GPGPU
\end{abstract}

\section{Introduction}

Small and inexpensive portable devices such as smartphones and tablet PCs have become adopted explosively into widespread use. The necessity for high-speed cryptographic technology for use with such devices has also increased. However, demand for high-performance encryption at high-end fields such as data centers holding enormous data, so-called "Big-data", continues growing too. These facts indicate that the field of security countermeasures is widely necessary. In fact, Intel and AMD have presented an AES-NI [1] instruction set that is implemented on a processor with an embedded cryptographic engine as a solution for high-speed encryption for a standard symmetric block cipher called Advanced Encryption Standard (AES) [2]. It drastically improves the speed of AES encryption by hardware acceleration. However, if AES is not used for a data encryption, then its high efficiency will not be valuable in the least, and the circuit area will have been wasted.

To date, heterogeneous many-core processors with a CPU-GPU (Graphics Processing Unit) integration architecture are becoming popular for their high-speed performance and low power consumption. A many-core architecture is scalable because the number of processor cores can be adjusted for use. Actually, computers embedding a GPGPU

\footnotetext{
Manuscript received January 11, 2013.

Manuscript revised May 16, 2013.

${ }^{\dagger}$ The authors are with the Department of Computer Science, National Defense Academy of Japan, Yokosuka-shi, 239-8686

a)E-mail: iwai @nda.ac.jp

DOI: 10.1587/transinf.E96.D.2575
} Japan. are widely employed from mobile phones e.g. A5 processor of iPhone, to PC and servers e.g. Intel Ivy and Sandy bridge. And also the needs to process cryptography on these devices exist. For this reason, accelerating cryptographic processing while using a many-core processor with scalable architecture is expected to constitute one solution for highspeed and scalable cryptographic processing [3]-[5]. Highspeed cryptographic processing accelerated by a many-core architecture such as that of GPGPUs will resolve problems that cannot be solved by AES-NI because those GPGPU accelerations based on a software implementation can accept any cryptographic algorithm. Moreover, such accelerators entail no extra hardware costs such as those associated with specialized circuits for cryptography because GPGPUs are embedded as a general purpose accelerator.

However another problem remains: difficulty of the program for GPGPU. Many factors are useful to exploit the performance of GPGPU for cipher applications. Particularly, problems such as optimizing the way of using various kinds of memory of GPGPU and considering computation granularity trouble many programmers. To address these problems, we suggest a special-purpose translator for symmetric block ciphers: HIgh-performance CRYptographic Program Translator (HiCrypt). HiCrypt generates optimized GPGPU programs written in CUDA [6] or OpenCL [7] from cipher programs written in a standard $\mathrm{C}$ language with directives. HiCrypt constitutes of a simple method to produce optimized cipher programs for GPGPU, because the translator requires annotations only of elements that are characteristic for cipher programs. In fact, HiCrypt requires no knowledge about a special feature nor an architecture of GPGPU. By supporting both of OpenCL and CUDA, HiCrypt provides the way to use various many-core accelerators to process cipher programs.

Moreover, HiCrypt targets not only users who need to process cryptography in high-speed but also developers and evaluators of cryptography. Although cipher algorithms are evaluated by its robustness, performance of which is also an important element for evaluation. Evaluators of cryptography such as National Institute of Standards and Technology (NIST), New European Schemes for Signatures, Integrity and Encryption (NESSIE) and Cryptography Research and Evaluation Committees (CRYPTREC) test processing speed of cryptography to select a standard and recommendation cryptography in fact. Processing speed on a general purpose processor and an embedded processor is evaluated for these purpose widely, however, performance accelerated by 
GPGPU is not adopted currently. HiCrypt will contribute for this situation by generating GPGPU's program from source code for evaluation of a general purpose CPU. And also HiCrypt will contribute to build a library for cryptography accelerated by GPGPUs. Some libraries for cipher program accelerated by GPGPU are now developed, however, these libraries are not employable condition because there are many platforms and many cipher algorithms.

The remainder of this paper is organized as follows. In the next section, we briefly introduce programming environments for GPGPU, CUDA and OpenCL. In Sect. 3, techniques for symmetric block ciphers on GPGPU are shown. In Sect. 4, details of HiCrypt directives are presented. In Sect. 5, design of the translator is shown. Implementation of the translator and its evaluation using five representative 128-bit symmetric block cipher programs, AES, Camellia [8], SC2000 [9], CIPHERUNICORN-A [10], and CLEFIA [11] are described in Sect. 6. Finally we explain our conclusion in Sect. 7.

\section{Programming Environment for GPGPU: CUDA and OpenCL}

To date, two industrial standard programming languages to make GPU programs are developed, CUDA and OpenCL. Each one provides both of a programming language and an abstract model for GPGPU. CUDA and OpenCL have many conceptual similarities but their terminologies are different. In this section, overview and important elements for cipher programs in each environment are shown.

CUDA is a programming tool for GPGPU computing provided by Nvidia Corp. CUDA provides an extended C language with type qualifier extensions for indicating data locality and a special function-call syntax for kernel invocation with specifying the parallelism. APIs for synchronization, initializing GPU and communication between GPU and CPU are also provided. In CUDA, a GPU of the graphics processor is hidden as a parallel computing architecture. CUDA architecture provides a structured hierarchical memory space and multiprocessor which consists of processors named streaming processors. Memory space consists of global memory, shared memory, constant memory, local memory, and registers. Multi-processor structure is hidden by thread execution model which provides single instruction multi-thread (SIMT) model. Each multi-processor executes 32 threads in parallel. CUDA exclusively supports NVIDIA GPUs and it can provide high-performance computing. On the other hand, OpenCL has published as a hardware independence programming framework for many-core environment including GPU. OpenCL is also published as an open standard specification. This standard defines an abstract device model which maps well to contemporary GPU architectures and a GPU programming model. It has a set of C language extensions called as "OpenCL C language" in the same manner as CUDA including APIs. In contrast to CUDA, OpenCL supports many devices provided by plural vendors, Apple, ARM, AMD, Nvidia, Intel and
Table 1 Comparison of terms of CUDA and OpenCL.

\begin{tabular}{c|c|c}
\hline \hline Category & CUDA term & OpenCL term \\
\hline Hardware & GPU & OpenCL Device \\
& Streaming processor & Processing element \\
& Streaming multiprocessor & Compute unit \\
\hline Execution & Grid & NDRange \\
& Block & Work-group \\
& Thread & Work-item \\
& Kernel & Kernel \\
& Stream & Command Queue \\
\hline Memory & Global memory & Global memory \\
& Constant memory & Constant memory \\
& Shared memory & Local memory \\
& Registers \& Local memory & Private memory \\
\hline
\end{tabular}

more. OpenCL architecture abstract consists of host computer and attached one or more compute devices. Each of which is comprised of one or more compute units with one or more processing elements. The OpenCL memory model describes a similar hierarchy to that of CUDA. Each compute device has global memory to which all work-items in work-groups, analogous with CUDA's threads and blocks, can read/write, local memory which is shared in a workgroup, private memory is private to each work-item and constant memory is same as CUDA's. Program written in each environment can be translated to each other. Some previous works compared CUDA with OpenCL and they also showed a comparison of terms used in each environment [12], [13]. Table 1 shows corresponding terms in both environments. Note that, CUDA terminology will be used in the rest of this paper, therefore readers can replace them with adequate OpenCL's word depending on their needs.

\section{Typical Implementation of High-Speed Symmetric Block Ciphers}

AES is adopted as an example of symmetric block cipher in this paper, although HiCrypt tries to translate many symmetric block ciphers. Moreover, some conditions are fixed to simplify the description herein. For example, the secret key size is fixed to 128-bit. The calculation time of an extended key, hereinafter referred to as "key", which is calculated from the secret key is not discussed because it is much smaller than the encryption time. Moreover, an encoding algorithm of AES is only discussed because decoding will apply the same technique as that shown in the encoding.

\subsection{AES}

AES is a symmetric block cipher introduced in 2001 by NIST [2]. Although 128-bit, 192-bit, and 256-bit key size can be selected, we discuss only 128-bit in this paper. Its algorithm of the 128-bit key defines 10-round processes. Each round includes four transformations: SubBytes, ShiftRows, MixColumns, and AddRoundKey. The final round differs slightly from the other rounds: it does not include MixColumns. In AES, a round process can be combined into a transformation simply using a look-up table called T-box 
and XOR operation [14]. Letting $a$ be the round input, which is divided into four inputs $a_{0}, a_{1}, a_{2}, a_{3}$, each of which consists of 32 bits, the round output $e$ is represented as

$$
e_{j}=T_{0}\left[a_{0, j}\right] \oplus T_{1}\left[a_{1, j+1}\right] \oplus T_{2}\left[a_{2, j+2}\right] \oplus T_{3}\left[a_{3, j+3}\right] \oplus k_{j}
$$

where $T_{0}, T_{1}, T_{2}$, and $T_{3}$ are look-up tables and $k_{j}$ is the $\mathrm{j}$-th column of a round key. This algorithm requires only four look-up table transformations and four XOR operations. Using a pre-computed look-up table such as T-box is valid for other symmetric block ciphers, but the look-up table requires more memory space. In fact, almost all software implementations of the block cipher use the look-up table.

Block ciphers have some modes - Electric Code Book (ECB), Cipher Block Chaining (CBC), CounTeR (CTR) and so on. Electronic Code Book (ECB), CountTeR (CTR), or Xor-encrypt-xor Tweakable code book mode with ciphertext Stealing (XTS) [15] are known as parallelizable modes in block cipher. ECB uses a single key applied to all plaintexts. CTR uses a key stream generated from a secret key and combined with plaintexts. In the CTR mode, the generation of the key stream is conducted in the same manner as ECB. In the XTS mode, plaintexts are encrypted using two ECB modes. Therefore, we discussed only ECB mode to evaluate the parallel processing of symmetric block ciphers.

\subsection{Optimization for Cipher Programs on GPGPU}

Some previous works had clarified which techniques are valid for the AES program with CUDA. Biagio et al. [16] described a study of the relations between the parallelizing granularity and its performance. The effect of the number of threads and file size are also shown. Iwai et al. [17] further discussed granularity and the variety of memory allocations for variables of cipher programs. It showed that efficient CUDA programs made a difference of more than 50 times in performance. Nishikawa et al. [18], [19] applied the technique shown in [17] to other ciphers to validate the effectiveness of it. The result showed that implemented ciphers, Camellia, CIPHERUNICORN-A, Misty, and SC2000 also exhibit high-performance. This section explains the optimization technique which is used by HiCrypt introduced in previous works.

\subsubsection{Granularity}

In 128-bit symmetric block ciphers, the input data are separated specifically to 128-bit block size and are then encrypted. Each 128-bit block can be processed by each thread in parallel. There is greater computation granularity such as 64-bit, 32-bit and 16-bit per thread. Even greater 128bit per thread granularity has the benefit of no synchronization instruction during parallel processing by multiple threads compared with the other granularity. For this reason, a HiCrypt translator set the computation granularity to 128 bit per threads in parallel encryption processing on GPUs.

\subsubsection{Memory Allocation}

Generally in optimized software implementation of symmetric block ciphers, the same look-up tables are referred multiple times. Their contents are not modified. Therefore, tables stored in shared memory with low access latency engender higher performance than those stored in global memory. If the cache mechanism works well, then constant memory will provide the same low latency as local memory. However, the look-up table operation of block cipher requires random access [17]. Therefore, constant memory should be a wrong selection to store for such look-up tables.

By contrast, the key can be stored in the shared memory or the constant memory because it is referred as common data by all threads and accessed in a regular pattern.

\subsubsection{Thread Management}

The following two solutions should exist to parallelize the encryption process of 128-bit block ciphers. One is to generate the same number of threads as the number of 128-bit blocks that construct a plaintext. Another one is to process several 128-bit blocks in a repetitive fashion by each thread. This technique shows $3-5 \%$ improvement over the first because look-up tables on the shared memory can be reused.

\section{Design of the Directives}

\subsection{Design Concept}

HiCrypt requires annotations for only characteristics of the cipher program, because HiCrypt is tailored to translating cipher programs. It means that HiCrypt requires neither parallelization nor optimization annotations. This is a major difference from other parallelization compiler/translators. Users can use HiCrypt easily, because all of "must directives" required by HiCrypt are essential elements for cipher programs such as variables of a plaintext and a ciphertext.

\subsection{Directives}

Directives of HiCrypt are specified with the \#pragma mechanism. The syntax of directive is displayed as described below.

line

\#pragma cryptcuda directive-name [clause] new-

Each directive starts with \#pragma cryptcuda. The remainder of the directive follows the $\mathrm{C}$ conventions for program. The format of this directive is similar to OpenACC [20] and so on. All of directives defined by HiCrypt are shown in Table 2 with their brief descriptions.

Directives of HiCrypt consist of directive groups of two kinds. The first one includes must directives, which annotate elements for characteristics of cipher programs. Another group consists of parameters for optimization and hints for a translator such as the size of the plaintext. 
Table 2 Brief descriptions of the directives.

\begin{tabular}{|c|c|c|}
\hline Annotate variables & For optimization & \\
\hline plaintext plaintext, pointer & $\operatorname{arch} \dagger$ & CUDA architecture \\
\hline ciphertext ciphertext, pointer & & version \\
\hline key, pointer & threadNum $\dagger$ & Number of threads \\
\hline look-up table & blockNum $\dagger$ & Number of blocks \\
\hline For functions & Others & \\
\hline kernelCall Earmark of kernel call & filesize $\dagger$ & Filesize of plaintext \\
\hline $\begin{array}{ll}\text { kernelFunc } & \begin{array}{l}\text { Kernel function definition } \\
\text { device }\end{array} \\
& \begin{array}{l}\text { Definition of function } \\
\text { called from kernel }\end{array}\end{array}$ & blocksize0fCipher† & Blocksize of cipher \\
\hline
\end{tabular}

Must directives, plaintext, ciphertext, key, table, kernelCall, and kernelFunc, are introduced here. Directives plaintext, ciphertext, key, and table annotate variables. They apply to an immediately following statement that is a variable declaration. The remaining two must directives, kernelCall and kernelFunc, annotate an encoding function call and its definition. kernelCall applies to an immediately following single statement that encodes function call so that the function can be offloaded to GPGPU. kernelFunc applies to an immediately following structured function definition block that is definition of function annotated kernelCall. The structured function definition block will be parallelized and translated to the OpenCL or CUDA program. The optional directive device provides a solution to call functions from an inner function, which is annotated as kernelFunc.

Other directives for optimization are arch, threadNum and blockNum. threadNum and blockNum provide a solution when users would like to optimize the number of thread and block manually, although HiCrypt has default numbers of them. Furthermore, arch defines an architecture of GPU such as CUDA's sm_20 and sm_13. It provides a hint for optimization to the translator.

Directives filesize and blocksize0fCipher provide a hint of a targeted cipher algorithm to the translator. If filesize and blocksize0fCipher are defined, then the translator will work reliably, but the translator will seek the file size of a plaintext and the block size of a cipher algorithm.

\subsection{Restrictions}

To use HiCrypt directives, some restrictions must be made on a program format. This section shows all restrictions required by the translator.

At the first brush, variables, which represent plaintext, ciphertext, and key, must be declared as a single-dimension array. Look-up tables annotated by table must be declared as single-dimension arrays too, although look-up tables for symmetric block cipher are sometimes declared as multidimension arrays. These restrictions are presented in Table 3. These pointer variables must have no aliases because the translator uses their names directly to generate a CUDA and OpenCL program.
Table 3 Restrictions: declaration of variables.

\begin{tabular}{l|l}
\hline \hline Variable type & Format \\
\hline Plaintext & type ${ }^{*}$ varname $;$ or type varname [size]; \\
\hline Ciphertext & type $*$ varname $;$ or type varname $[$ size $] ;$ \\
\hline Key & type $*$ varname or type varname[size]; \\
\hline Tables & type varname $[$ size $]=\left\{{ }^{*}\right.$ statically initialize $\left.* /\right\}$ \\
\hline
\end{tabular}

There are two additional restrictions. The first is a restriction on order and members of argument of the function definition, shown as below:

void function-name(integertype *plaintextpointer, integertype *ciphertextpointer, integertype *key, integertype numberofblocks);

Types of the dummy arguments except numberofblocks accept any integer pointer type, such as unsigned char*, int* and long*. The type of the plaintextpointer and $\mathrm{ci}^{-}$ phertextpointer must be the same. Moreover, dummy arguments plaintextpointer, ciphertextpointer and key must have no aliases. Another restriction is format of the "for" loop, which encodes a cipher block repetitively in an encoding function annotated by the directive. The loop must be written according to the format shown below:

$$
\begin{aligned}
& \text { for }(\text { counter }=0 ; \text { counter }<\text { numberofblock; } \\
& \text { counter }++ \text {, plaintextpointer }+=\text { blocksize, } \\
& \text { ciphertextpointer }+=\text { blocksize }) .
\end{aligned}
$$

The parameter numberofblock represents the number of plaintext blocks and blocksize represents the block size of the block cipher. For example, the block size of AES is described as "16" in byte size or "4" in word (32-bit) size. HiCrypt accepts any type of variables and there is no need to care for it, because HiCrypt can parallelize a formatted for loop shown above by the simple dividing. However it is better to use these variables' unit in coherent for safe programming. Also HiCrypt applies simple type-checking for these variables as an assistance for users.

These restrictions must be accepted to use HiCrypt. However these restrictions are reasonable for users because many optimized programs for symmetric ciphers are written similarly. To explain details of these directives and the behavior of the translator, a base code for AES with HiCrypt's directives is shown in Fig. 1 as an example. This code shows only important statements and structures of the program. 


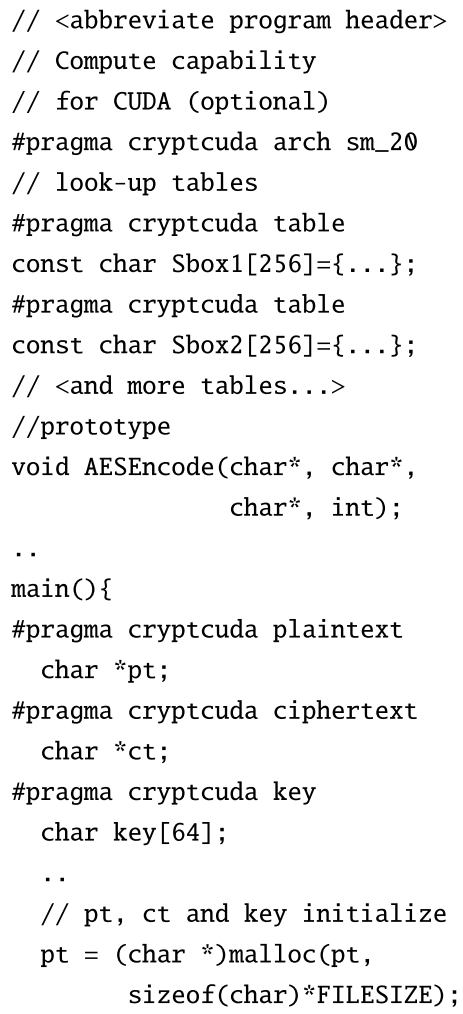

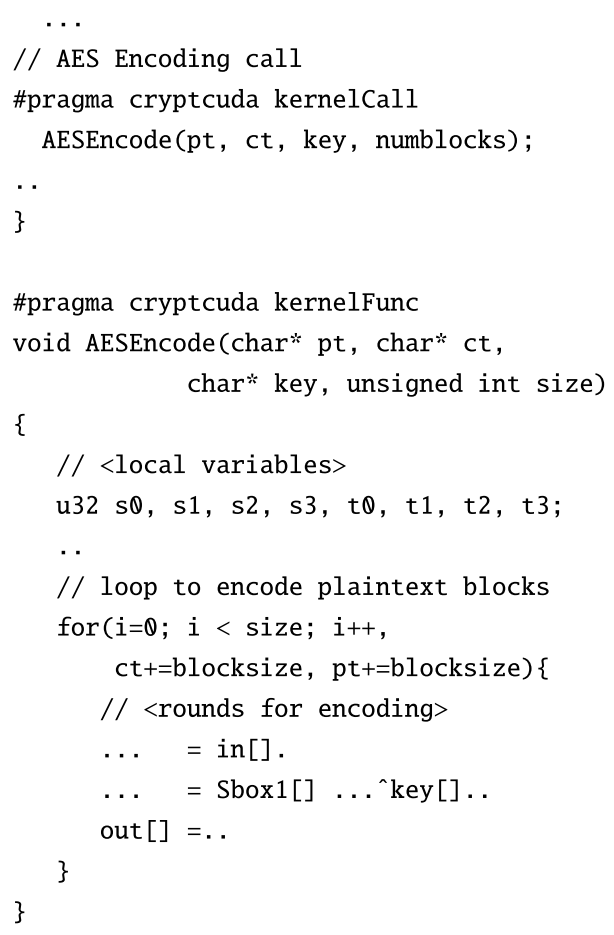

Fig. 1 Base code of AES.

\section{Design of the Translator}

\subsection{Translation Overview}

Two groups of programs, host program and kernel program are generated by HiCrypt in both of OpenCL and CUDA environment. Additional statements, which consist of qualifiers, new variables for GPGPU, data transfer and allocation APIs, GPGPU initialization, and kernel call are generated into the host program. Also generating statements, which are qualifiers, new variables and initialization to use the shared memory, and restructuring of encoding loop for parallelizing are applied for kernel program.

\subsection{The flow of Translation}

An implemented translator described in Sect. 6 scans a source program twice as shown in Fig. 2. On the first pass, it finds all directives and annotated elements in the source program. Then it stores them in its working memory, called a directive table. A symbol table which stores all symbols in source program is also built simultaneously. Contents of the directive table for AES are shown in Table 4 for example.

On the second pass, it translates statements of the source program annotated by the directives to an optimized CUDA/OpenCL program. The transformation technique for the host program consists of primary four parts, "Creating new variables", "Generating code for device initialization",

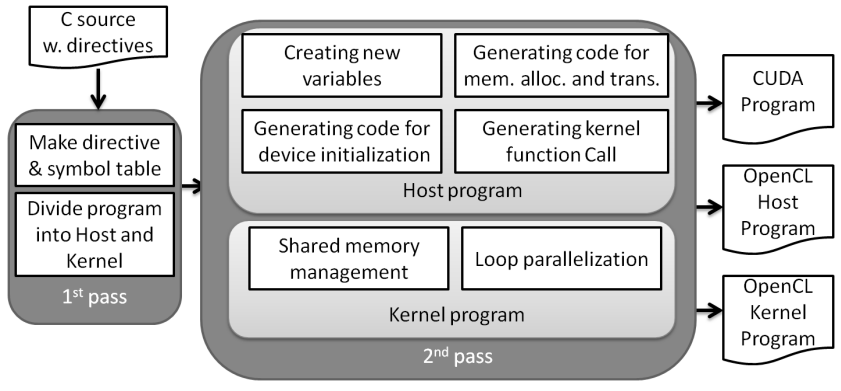

Fig. 2 The flow of translation.

Table 4 Directive table for AES base code.

\begin{tabular}{llllll}
\hline \hline Directive & ID & Type & Size & Line & Filename \\
\hline plaintext & pt & char* & FILESIZE & 593 & AESCore.c \\
\hline ciphertext & ct & char* & FILESIZE & 595 & AESCore.c \\
\hline key & e_key & char* & 64 & 591 & AESCore.c \\
\hline table & Sbox1 & uint32 [] & 256 & 52 & AESCore.c \\
\hline table & Sbox2 & uint32 [] & 256 & 187 & AESCore.c \\
\hline table & Sbox3 & uint32 [] & 256 & 321 & AESCore.c \\
\hline table & Sbox4 & uint32 [] & 256 & 454 & AESCore.c \\
\hline kernelCall & AESEncode & NA & NA & 631 & AESCore.c \\
\hline kernelFunc & AESEncode & NA & NA & 659 & AESCore.c
\end{tabular}

"Generating code for allocation and transfer" and "Generating kernel function call". Moreover, translation of kernel program consists of "shared memory management" and "Loop parallelization". Finally, they are outputted as a single file in case of CUDA but as discrete files in case of 
Table 5 Recommended combinations for variables and memory hierarchy.

\begin{tabular}{c|c}
\hline \hline Variable & Memory \\
\hline plaintext and ciphertext & Global Memory \\
\hline Key & Constant memory or Shared Memory \\
\hline Table & Shared memory \\
\hline
\end{tabular}

Table 6 Qualifier for variables for CUDA and OpenCL.

\begin{tabular}{l|l|l}
\hline \hline Type & CUDA & OpenCL \\
\hline pointer for global memory & - & cl_mem \\
pointer for constant memory & -_constant_- & --constant \\
pointer for shared memory & -_shared_-_ & _-local \\
kernel function prototype & -_global_- & _-_kernel \\
\hline
\end{tabular}

OpenCL.

\subsection{Translation of Host Program}

\subsubsection{Creating New Variables}

HiCrypt creates new variables which contain data used on the GPU at the beginning. In cipher programs, they are plaintext, ciphertext, key and table. These elements are annotated by the HiCrypt directive. Figure 1 shows these annotated variables pt, ct, key, and S_box. HiCrypt makes these new variables with unique names which correspond with the memory on which each variable is stored. Each variable must be placed in a correct hierarchy of memory according to previous work. The best combination of variables and memory hierarchy which was shown in a previous work are summarize in Table 5. HiCrypt allocate these variables to adequate places according to the combination of variables and memory using memory hierarchy qualifiers. Qualifiers for each variable to allocate each memory are shown in Table 6 . The translator creates new variables with added prefix "d_" to the basename to be unique ${ }^{\dagger}$, to allocate pt and ct shown in Fig. 1 on the global memory. Similarly, new variables to which are added prefix "c" " to the basename to allocate variables annotated by key and table in the constant memory are created ${ }^{\dagger}$. Their declaration is also created in front of a main() function.

Tables must be allocated on the shared memory of the GPU. However, data cannot be sent from CPU directly to the shared memory. Tables need not always be allocated on constant memory. However, the current version of the translator allocates them on the constant memory first. Furthermore, allocating a variable for a key on the shared memory is valid. The key can be allocated on the shared memory or constant memory because each allocation does not make a difference for their performance [17].

Some more variables to control OpenCL program are generated to keep information device id, kernel program and more, although CUDA program does not require them. These variables are generated as a simple constant of strings. Figure 3 portrays a generated program by the translator. Variables created by the translator are placed at lines 9-16 in Fig. 3.

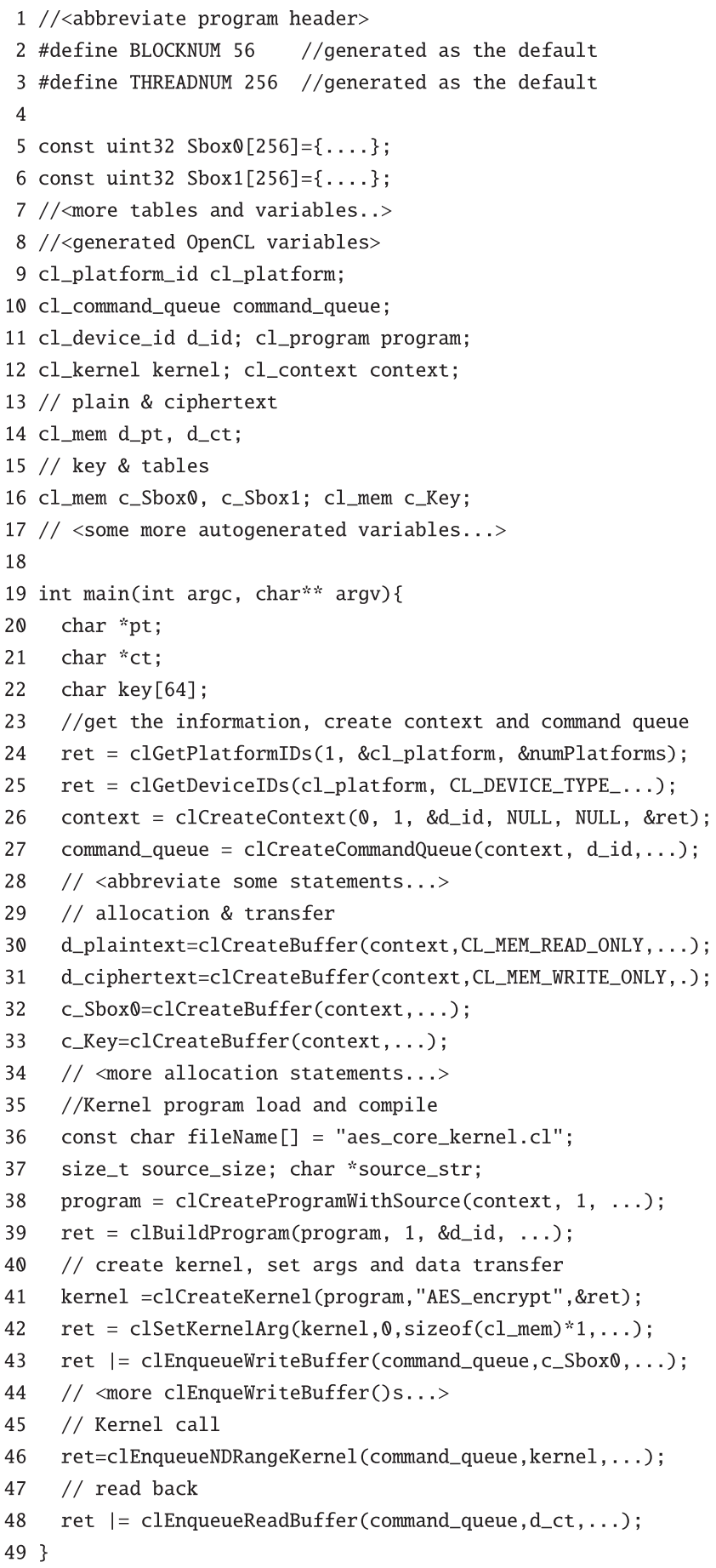

Fig. 3 Generated OpenCL program including new variable declaration, allocation, transfer and kernel call except kernel function definition.

\subsubsection{Generating Code for Device Initialization}

When the translator finds a kernelcall directive in main() function, statements for initializing device are generated. Only CUDA_Init () is created in case of CUDA program. On the other hand, some procedures such as getting device information and making command queues to make 
Table 8 Generated APIs for data allocation and transfer.

\begin{tabular}{l|l|l|l}
\hline \hline & CUDA & OpenCL & Allocated or transferred variables \\
\hline Device memory allocation & cudaMalloc() & - & clCreateBuffer() \\
Constant memory allocation & clCreateBuffer() & key, tables (only OpenCL) \\
Data transfer (constant memory) & cudaMemcpyToSymbol() & clEnqueueWriteBuffer() & key, tables \\
Data transfer (host to device) & cudaMemcpy() & clEnqueueWriteBuffer() & plaintext \\
Data transfer (device to host) & cudaMemcpy() & clEnqueueReadBuffer() & ciphertext \\
\hline
\end{tabular}

Table 7 Generated APIs for initialize.

\begin{tabular}{l|c|l}
\hline \hline Type & CUDA & OpenCL \\
\hline Initialize & CUDA_Init() & \multicolumn{1}{c}{-} \\
\hline $\begin{array}{c}\text { Getting device } \\
\text { information }\end{array}$ & - & $\begin{array}{l}\text { clGetPlatformIDs() } \\
\text { clGetDeviceIDs() }\end{array}$ \\
\hline $\begin{array}{c}\text { Making command } \\
\text { queues }\end{array}$ & - & $\begin{array}{l}\text { clCreateContext(), } \\
\text { clCreateCommandQueue() }\end{array}$ \\
\hline
\end{tabular}

program are required by OpenCL. These initializing statements are simply created as a constant strings value. Example of created APIs in this stage is shown in Table 7, but some more APIs may be generated for initialization. Initialization created by the translator is placed at lines $24-27$ in Fig. 3.

\subsubsection{Generating Code for Allocation and Transfer}

APIs for the device memory allocation and data transfer are generated after device initialization code. Generated APIs for an allocation and a transfer are shown in Table 8. Firstly to allocate a ciphertext and a plaintext on the global memory, two cudaMalloc() or clCreateBuffer() are generated. Also clCreateBuffer() is required for OpenCL to use constant memory for key and tables, although CUDA does not require. Then data transfer codes for all created variables are generated, cudaMemcpy and cudaMemcpyToSymbol for CUDA, and clEnqueueWriteBuffer() for OpenCL. Their parameters, variable name, its type, and size are generated by automatically based on information stored in the directive table. Then, to receive ciphertext processed on GPU, cudaMemcpy () or clEnqueueReadBuffer are generated after the encoding function call. These generated codes are shown in Fig. 3 at lines 30-34, 43-44, 48.

\subsubsection{Generating Kernel Function Call}

OpenCL requires more procedure to create kernel call compared with CUDA program. In case of OpenCL, one clCreateKernel() to create kernel object, same number of clSetKernelArg() as arguments of kernel function, and clEnqueueNDRandgeKernel() to invoke kernel are generated. Moreover to compile kernel program (called on-line compile), clCreateProgramWithSource() and clBuildProgram() are created for OpenCL program. Meanwhile, simply two dim3 type variables to set the threads and the blocks, and function call to invoke kernel in original format are generated for CUDA.

The number of threads and blocks are given by directives as threadNum and blockNum. If they are not specified,
Table 9 Elements attached in kernel function definition.

\begin{tabular}{l|l|l}
\hline \hline Element & CUDA & OpenCL \\
\hline $\begin{array}{l}\text { Kernel function qualifier } \\
\text { shared memory qualifier for vars. }\end{array}$ & $\begin{array}{l}\text {-_global_-_ } \\
\text { shared_-- }\end{array}$ & $\begin{array}{l}\text {-_kernel } \\
\text {-_local }\end{array}$ \\
\hline shared memory vars. & \multicolumn{2}{|c}{ prefix "s_" added to } \\
& \multicolumn{2}{|c}{ variables annotated with table } \\
\hline $\begin{array}{l}\text { API to get the block id } \\
\text { API to get the thread id }\end{array}$ & $\begin{array}{l}\text { blockIdx.x } \\
\text { threadIdx.x }\end{array}$ & $\begin{array}{l}\text { get_global_id() } \\
\text { get_local_id() }\end{array}$ \\
\hline thread synchronization & syncthreads() & barrier() \\
\hline
\end{tabular}

then the translator will attempt to calculate the best numbers according to an architecture of GPU. Default value of the blockNum is 28 or its multiples for Tesla C2050 currently. Default value of the threadNum is 256 or its multiples. To minimize a loss of performance attributable to padding in case of odd size of plaintext, it is better to set the number of threads and blocks as not too numerous. In contrast, to obtain higher performance, a certain number of blocks and threads are necessary. The kernel call including relevant API is shown in Fig. 3 at lines 36-42, 46.

\subsection{Translation of Kernel Program}

Kernel program is generated through translating encoding function. It is another important task for the translator. This task consists of two parts: shared memory management and loop parallelization.

\subsubsection{Shared Memory Management}

When the translator meets up with the directive kernelFunc, the translator adds qualifier _-global_- or --global to the line before the kernel function definition. In case of OpenCL, dummy arguments which consist of pointer for tables on the constant memory and tables on the shared memory are also added to original function. Then, new shared variables to store data of tables with a prefix "s_" added to variables annotated with table are created ${ }^{\dagger}$. Declarations of new variables are placed at the beginning of the body of the kernel function definition with keyword _-_shared__or __local. Qualifiers used by the kernel program translation in CUDA and OpenCL are shown in Table 9. A variable annotated with table is called "table" hereinafter and a new variable allocated on the shared memory is hereinafter called an "s_table". A table variable allocated on the constant memory is called a "c_table".

To fill up each s_table with the content of each table that

$\dagger$ If created variable name was not unique, HiCrypt renames it to be unique. 
Table 11 Summary of the cipher specifications.

\begin{tabular}{ccccc}
\hline \hline Cipher & Structure & \# of tables & Size of tables & \# of rounds* \\
\hline AES & SPN & 4 & 1 Kbyte/table & 10 \\
Camellia & Feistel & 4 & 1 Kbyte/table & 18 \\
SC2000 & Hybrid & $3 * *$ & 8 Kbyte/table** & 7 \\
CIPHERU. & Feistel & 1 & 1 Kbyte/table & 16 \\
CLEFIA & Feistel & 8 & 1 Kbyte/table & 18 \\
*in case of 128-bit key length. & & \\
**Optimized as speed. \# of tables and table size are selectable in SC2000.
\end{tabular}

have achieved the best throughput in the symmetric block ciphers currently. [19]. CLEFIA is adopted as a ISO/IEC 29192 light weight cryptography which is a new category of cryptography. In this evaluation, the length of the key is fixed at 128-bit length for all cipher programs. Number of round and table are different each other. Table 11 presents a summary of the specification of five ciphers.

\subsection{Creating a Source Program with Directives}

Source codes of AES and Camellia are made from OpenSSL/TLS source code. Particularly, primary encoding codes are almost a direct quote from it. By contrast, SC2000, CIPHERUNICORN-A, and CLEFIA are implemented based on the specification [9]-[11] by one author of this paper. All programs are implemented with a restricted format shown as before. The directives, plaintext, ciphertext, key, table, kernelCall and kernelFunc are used. Therefore, the format of these programs is the same as a base code of AES shown in Fig. 1. The directive device is annotated to call the function in the kernel function in case of SC2000. All programs are implemented using look-up tables to speed up encryption, although some of ciphers have a quite different structure. Implementations without look-up table, which provide 10 times or more performance degradation compared with the look-up table based implementation, are not discussed because the purpose of HiCrypt is to achieve high performance cipher processing. The bitslice implementation, one of the high-performance implementation, is not employed by this paper because a bitslice implementation is not always true that it exerts better performance than look-up table based implementation [22]. Moreover, there is need to make up a difficult program design such as SIMD-like programming using general programming language such as the $\mathrm{C}$ in case of bitslice implementation. However all programs have different data randomize code in the source code, directives are easily inserted to all five ciphers. Because annotated variables are distinguished for all symmetric block cipher programs, making a program with HiCrypt directives is easy for programmers.

\subsection{Performance Result}

The translator could successfully generate OpenCL and CUDA programs for all five ciphers. Performances of generated programs and hand optimized programs are presented in Table 12. The hand optimized program is same as the
Table 12 Throughputs of AES, Camellia, SC2000, CIPHERUNICORNA, and CLEFIA. HiCrypt: generated program by HiCrypt. Hand: hand optimized program.

\begin{tabular}{|c|c|c|c|c|}
\hline Algorithm & Platform & Program & $\mathrm{Gb} / \mathrm{sec}$ & Cycles/byte \\
\hline \multirow[t]{5}{*}{ AES } & \multirow{2}{*}{ CUDA } & HiCrypt & 53.2 & 0.17 \\
\hline & & Hand & 53.3 & 0.17 \\
\hline & \multirow[t]{2}{*}{ OpenCL } & HiCrypt & 50.6 & 0.18 \\
\hline & & Hand & 50.6 & 0.18 \\
\hline & CPU & - & 1.3 & 16.58 \\
\hline \multirow[t]{5}{*}{ Camellia } & \multirow[t]{2}{*}{ CUDA } & HiCrypt & 54.9 & 0.17 \\
\hline & & Hand & 54.9 & 0.17 \\
\hline & \multirow[t]{2}{*}{ OpenCL } & HiCrypt & 52.6 & 0.17 \\
\hline & & Hand & 52.6 & 0.17 \\
\hline & CPU & - & 1.0 & 22.1 \\
\hline \multirow[t]{5}{*}{ SC2000 } & \multirow[t]{2}{*}{ CUDA } & HiCrypt & 83.0 & 0.11 \\
\hline & & Hand & 83.0 & 0.11 \\
\hline & \multirow[t]{2}{*}{ OpenCL } & HiCrypt & 71.4 & 0.13 \\
\hline & & Hand & 71.4 & 0.13 \\
\hline & $\mathrm{CPU}$ & - & 1.1 & 19.89 \\
\hline \multirow[t]{5}{*}{ CIPHERU. } & \multirow[t]{2}{*}{ CUDA } & HiCrypt & 25.0 & 0.37 \\
\hline & & Hand & 25.0 & 0.37 \\
\hline & \multirow[t]{2}{*}{ OpenCL } & HiCrypt & 25.0 & 0.37 \\
\hline & & Hand & 25.0 & 0.37 \\
\hline & $\mathrm{CPU}$ & - & 0.2 & 99.47 \\
\hline \multirow[t]{5}{*}{ CLEFIA } & \multirow[t]{2}{*}{ CUDA } & HiCrypt & 53.7 & 0.17 \\
\hline & & Hand & 53.7 & 0.17 \\
\hline & \multirow[t]{2}{*}{ OpenCL } & HiCrypt & 51.3 & 0.18 \\
\hline & & Hand & 51.3 & 0.18 \\
\hline & CPU & - & 1.3 & 17.09 \\
\hline
\end{tabular}

program which is shown in our previous work [19] except OpenCL's. OpenCL programs are manually written according to CUDA's. HiCrypt is designed to generate similar programs with our previous work, therefore, performance results of hand optimized programs and programs generated by HiCrypt shown in Table 12 are almost identical. In case of CLEFIA, it is implemented for hand optimized program in the same mannar as other cipher programs. To evaluate their performance in cases near peak performance, each parameter is determined: the number of threads in a thread block is 512, the number of thread blocks is 56 and the size of plaintext is 10 MBytes. OpenCL programs exhibit around $0 \%-15 \%$ performance reduction. That is a well known fact, a current version of OpenCL program sometimes shows performance reduction compared with the CUDA program because of OpenCL compiler's immaturity [12]. As a result, HiCrypt succeed to generate optimized parallelized code for cipher programs in the both of environments, CUDA and OpenCL. Table 12 does not show a comparison of performance of CPU and GPU, although each performance is shown. CPU's throughput shows a validity of the base codes. To compare performance of GPU and CPU, there is a need to consider about a device cost, power consumption, CPU's programming environment, and more. Next, a performance comparison with several previous works for AES and Camellia is shown in Table 13. Performance of hand optimized AES and Camellia program by GTX285 from our previous work [19] is also shown. No related work for CIPHERUNICORN-A, Hierocrypt-3, SC2000, nor CLEFIA using GPGPU is described in the literature without our previous work. 
Table 13 Performance results of previous works.

\begin{tabular}{l|c|c|c|c}
\hline \hline Reference & Framework & Algorithm & Device & Gb/sec \\
\hline Nishikawa et al. [19] & CUDA & AES & GTX285 & 37.8 \\
\hline Nishikawa et al. [19] & CUDA & Camellia & GTX285 & 38.0 \\
\hline Osvik et al. [25] & CUDA & AES & GTX295*3 & $30.9^{* 4}$ \\
\hline Li et al. [23] & CUDA & AES & C2050 & 60 \\
\hline Gilger et al. [26] & CUDA & AES & GTX295*3 & 29.6 \\
\hline Gilger et al. [26] & OpenCL & AES & GTX295*3 & 25.4 \\
\hline Gilger et al. [26] & CUDA & Camellia & GTX295*3 & 26.3 \\
\hline Gilger et al. [26] & OpenCL & Camellia & GTX295*3 & 26.3 \\
\hline *3 They use only 1 of 2 chips of GPU (240 cores) \\
${ }^{* 4}$ Including data transfer time
\end{tabular}

Qinjian $\mathrm{Li}$ et al.[23] implemented AES on Nvidia C2050 using CUDA and achieved $60 \mathrm{~Gb} / \mathrm{sec}$ throughput by reordering plaintext data. A quantitative cost of reordering was not shown. This implementation looks similar to our hand optimized implementation and it refers our previous work [24]. Therefore, generated AES program by HiCrypt will have the same quality as this work by considering to the cost of reordering.

Osvik et al. [25] and Gilger et al. [26] showed AES implementation on Nvidia GTX295 and both previous works achieved around $30 \mathrm{~Gb} / \mathrm{sec}$ used half of GTX295. The half of GTX295 contains the same number of cores as GTX285 but GTX295's core clock (576MHz) is slower than GTX285's (648MHz). If these programs had processed by C2050, throughput would be estimated roughly $53.2 \mathrm{~Gb} / \mathrm{sec}$ by calculating with the number of cores and the clock speed though, it is difficult to compare performance between different architectures. Anyway, a generated code by HiCrypt has good enough quality since the throughput of program generated by HiCrypt nears estimated throughput of previous works. Note that Osvik's throughput includes data transfer, so there are possibilities that the data transfer program was implemented in a skillful manner to hide the cost of the data transfer or AES processing was implemented faster.

Gilger et al. also showed the performance of Camellia on Nvidia GTX295 and achieved $26.3 \mathrm{~Gb} / \mathrm{sec}$. Converting this throughput to C2050's, $45.5 \mathrm{~Gb} / \mathrm{sec}$ is led. This throughput is slower than the throughput of Camellia generated by HiCrypt.

\subsection{Translation Result}

As shown before, the programs generated by HiCrypt achieved almost identical performance as those of hand optimized programs. Therefore, the generated programs seem almost identical to the hand optimized program, except for the order of the API call and the places of declarations of variables.

The usage of OpenCL and CUDA is quite similar to one another, but the amount of the created statement for OpenCL is much greater than CUDA's. Total number of lines of each cipher program is shown in Table 14. Note that, these lines include an encoding program, directives, variable declarations, main function and more, except a definition
Table 14 Increase of amount of lines of generated program by HiCrypt without lines for a definition of table of S-boxes.

\begin{tabular}{c|c|c|c|c|c}
\hline \hline Algorithm & AES & Camellia & SC2000 & CIPHERU & CLEFIA \\
\hline \# of lines (input) & 307 & 215 & 213 & 341 & 230 \\
\hline \# of lines (OpenCL) & 504 & 414 & 441 & 521 & 455 \\
\hline Ratio & 1.64 & 1.93 & 2.07 & 1.53 & 1.98 \\
\hline \# of lines (CUDA) & 377 & 285 & 282 & 396 & 319 \\
\hline Ratio & 1.23 & 1.32 & 1.32 & 1.16 & 1.39 \\
\hline
\end{tabular}

of look-up table. Increase of amount of lines of the programs is around $160 \%$ to $200 \%$ in case of OpenCL, around $116 \%$ to $140 \%$ in case of CUDA compared to the source code with the directives. The number of generated statements for OpenCL program is about three times more than CUDA's one. Only around six to ten extra lines are required for HiCrypt to translate with only must directives, because six must directives shown before consist of one table directive and the others. Same number of the table directive as the number of tables contained target cipher programs must be annotated. As a result, HiCrypt can reduce a burden to write considerable additional CUDA/OpenCL code by inserting only around ten directives. Furthermore, All five programs which are written in a standard $\mathrm{C}$ program with HiCrypt directives have been compiled using a standard $\mathrm{C}$ compiler that ignored directives of HiCrypt.

HiCrypt has two more features, allowing nested loops and device function that calls an inner kernel function. Symmetric cipher algorithms consist of rounds which are repeated in the encoding process. To repeat rounds, a loop structure such as a "for" or "while" is used in an encoding function. In other words, loops for encoding to cipher blocks have a nested loop structure. Moreover, some datarandomize operations in rounds are defined as function calls to make a readable program. HiCrypt provides the directive device to support such a function. Results show that HiCrypt can translate such nested loops and device function calls. In contrast, general purpose automatic parallelization compilers for accelerator including GPGPU have been appearing recently, such as OpenACC [20], PGI Accelerator [27], and CAPS HMPP [28]. Moreover, academic suggestions to assist to make up OpenCL and CUDA programs have also been presented [29]-[31]. HiCrypt differs from them in their purpose, which is specialized for symmetric block ciphers. To make a small target, HiCrypt generated highly optimized CUDA programs from symmetric block cipher programs. As a future work, we would like to develop other features of HiCrypt such as automatic translation for public key cryptography. Furthermore, syntax analysis will be implemented to HiCrypt to relax its restriction.

\section{Conclusion}

This paper described a new translator, HiCrypt, for symmetric block ciphers. HiCrypt can generate optimized OpenCL and CUDA programs from symmetric block cipher programs written in a standard $\mathrm{C}$ language with HiCrypt directives. Usage of directives is simple for cipher pro- 
grammers because directives require annotation of some elements that are characteristics of the cipher programs. To evaluate HiCrypt, we implemented the translator and evaluated its performance through translating of five symmetric block cipher programs, AES, Camellia, SC2000, CIPHERUNICORN-A and CLEFIA with directives. Results show that the translator could generate optimized OpenCL and CUDA programs that exhibit almost identical performance as hand optimized programs for all five cipher programs. Moreover, the translator accepts a nested loops as a target loop and a calling function in the target loop.

Ciphers are evaluated by not only its strength but also its performance. Although the heterogeneous multiprocessors that embed a GPU have become widely used, they will be able to accelerate ciphers. Therefore, we are suggesting to evaluate cipher's performance by GPGPU. For this reason, HiCrypt will contribute to the development of new symmetric block ciphers and evaluating their performance.

\section{Acknowledgements}

This work is supported by Grant-in-Aid for Young Scientists B (25871223).

\section{References}

[1] Intel Corps., "Securing the Enterprise with Intel AES-NI," http://www.intel.com/content/dam/doc/white-paper/ enterprise-security-aes-ni-white-paper.pdf, 2010.

[2] National Institute of Standards and Technology (NIST), FIPS-197 Advanced Encryption Standard (AES), 2001.

[3] S.A. Manavski, "CUDA compatible gpu as an efficient hardware accelerator for AES cryptography," IEEE International Conference on Signal Processing and Communication, ICSPC, pp.65-66, 2007.

[4] G. Agosta, A. Barenghi, F.D. Santis, A.D. Biagio, and G. Pelosi, "Fast disk encryption through GPGPU acceleration," PDCAT, pp.102-109, 2009.

[5] M. Alomari, "A framework for GPU-accelerated AES-XTS encryption in mobile devices," IEEE Region 10 Conference TENCON 2011, pp.144-148, 2011

[6] "NVIDIA CUDA," http://developer.nvidia.com/object/cuda.html, 2009.

[7] K. Group, "Open compute language," http://www.khronos.org/

[8] K. Aoki, T. Ichikawa, M. Kanda, M. Matsui, S. Moriai, J. Nakajima, and T. Tokita, "Specification of Camellia —a 128-bit Block Cipher Version 2.0," 2001.

[9] T. Shimoyama, H. Yanami, K. Yokoyama, M. Takenaka, K. Itoh, J. Yajima, N. Torii, and H. Tanaka, "The block cipher SC2000," Revised Papers from the 8th International Workshop on Fast Software Encryption, FSE '01, London, UK, pp.312-327, 2002.

[10] NEC Corp., "Cryptographic specifications CIPHERUNICORN-A," 2002.

[11] M. Katagi and S. Moriai, "The 128-bit blockcipher CLEFIA (RFC6114)," 2011.

[12] M. Harvey and G.D. Fabritiis, "Swan: A tool for porting CUDA programs to OpenCL," Computer Physics Communications, vol.182, no.4, pp.1093-1099, 2011.

[13] P. Du, R. Weber, P. Luszczek, S. Tomov, G. Peterson, and J. Dongarra, "From CUDA to OpenCL: Towards a performanceportable solution for multi-platform GPU programming," Parallel Computing, vol.38, no.8, pp.391-407, 2012.

[14] J. Daemen and V. Rijmen, The Design of Rijndael: AES - The Advanced Encryption Standard, Springer Verlag, Berlin, Heidelberg,
New York, 2002.

[15] The IEEE Security in Storage Working Group, "XTS block cipherbased mode (XEX-based tweaked-codebook mode with ciphertext stealing)." http://siswg.net/

[16] A.D. Biagio, A. Barenghi, G. Agosta, and G. Pelosi, "Design of a parallel AES for graphics hardware using the CUDA framework," IPDPS, pp.1-8, 2009.

[17] K. Iwai, N. Nishikawa, and T. Kurokawa, "Acceleration of AES encryption on CUDA GPU," Int. J. Networking and Computing, vol.2, no.1, pp.131-145, 2012.

[18] N. Nishikawa, K. Iwai, and T. Kurokawa, "High-Performance Symmetric Block Ciphers on Multicore CPU and GPUs," Int. J. Networking and Computing, vol.2, no.2, pp.251-268, 2012.

[19] N. Nishikawa, K. Iwai, and T. Kurokawa, "Implementation of Symmetric Block Ciphers Using GPGPU," Proc. 7th International Conference on Information Warfare and Security, pp.222-232, 2012.

[20] "The OpenACC Application Programming Interface," White Paper, 2011.

[21] "New European Schemes for Signatures, Integrity, and Encryption," https://www.cosic.esat.kuleuven.be/nessie/

[22] M. Matsui and J. Nakajima, "On the power of bitslice implementation on intel core2 processor," Cryptographic Hardware and Embedded Systems - CHES 2007, LNCS 4727, pp.121-134, 2007.

[23] Q. Li, C. Zhong, K. Zhao, X. Mei, and X. Chu, "Implementation and analysis of aes encryption on gpu," 2012 IEEE 14th Int'l Conf. on High Performance Computing and Communication (HPCC) \& 2012 IEEE 9th Int'l Conf. Embedded Software and Systems (ICESS), pp.843-848, June 2012.

[24] K. Iwai, T. Kurokawa, and N. Nisikawa, "AES encryption implementation on CUDA GPU and its analysis," 2010 First International Conference on Networking and Computing, pp.209-214, 2010.

[25] D.A. Osvik, J.W. Bos, D. Stefan, and D. Canright, "Fast software aes encryption," 17th International Wrokshop, Fast Software Encryption, pp.75-93, Feb. 2010.

[26] J. Gilger, J. Barnickel, and U. Meyer, "Gpu-acceleration of block ciphers in the openssl cryptographic library," 15th International Conference, Information Security (ISC 2012), LNCS 7483, pp.338-353, 2012.

[27] "PGI Accelerator Programming Model for Fortran \& C," White Paper, 2010.

[28] R. Dolbeau, S. Bihan, and F. Bodin, "HMPP: A hybrid multi-core parallel programming environment," Proc. Workshop on General Purpose Processing on Graphics Processing Units, GPGPU'2007, pp.334-350, 2007.

[29] S.Z. Ueng, M. Lathara, S.S. Baghsorkhi, and W. mei W. Hwu, "CUDA-Lite: Reducing GPU Programming Complexity," LCPC, ed. J.N. Amaral, Lecture Notes in Computer Science, vol.5335, pp.1-15, 2008.

[30] M. Grossman, A.S. Sbîrlea, Z. Budimlic, and V. Sarkar, "CnCCUDA: Declarative Programming for GPUs," LCPC, ed. K.D. Cooper, J.M. Mellor-Crummey, and V. Sarkar, Lecture Notes in Computer Science, vol.6548, pp.230-245, 2010.

[31] S. Lee and R. Eigenmann, "OpenMPC: Extended OpenMP Programming and Tuning for GPUs," Proc. 2010 ACM/IEEE Conference on Supercomputing, pp.1-11, 2010. 


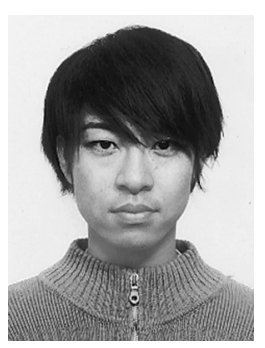

Keisuke Iwai completed the doctoral program in Department of C.S., Keio University in 1998 and fulfilled credit requirement in 2001. He holds a D.Eng. degree. He is now a lecturer in the Department of computer science, National Defense Academy of Japan. He is engaged in research on automatic parallel compilers and multi-processor systems.

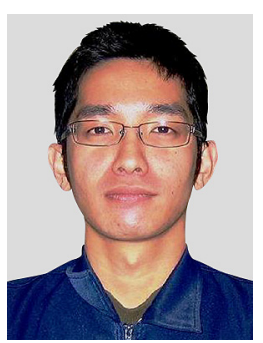

Naoki Nishikawa received his Master degree from the Department of C.S., National Defense Academy of Japan, in 2010. From 2010 to 2011 , he was affiliated with the Technical Research and Development Institute. He is now a $\mathrm{Ph} . \mathrm{D}$ student at National Defense Academy. He is interested in GPGPU and its application, cryptography, and SIMD processing.

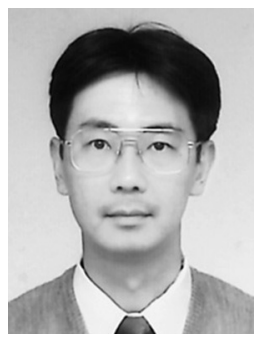

Takakazu Kurokawa received his B.S. degree from the Department of E.E, Keio University, in 1983 and completed the doctoral program in 1988. He holds a D.Eng. Degree. He is now a professor in the Department of C.S., National Defense Academy of Japan. He is engaged in research on dedicated computers, cryptography. 